\title{
Ein Symposium wirft Fragen auf
}

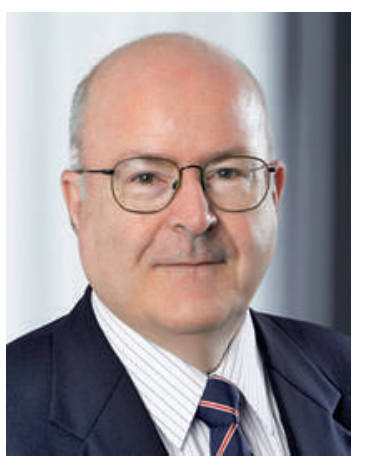

Das Schweizerische Institut für ärztliche Weiter- und Fortbildung SIWF hat kürzlich sein erstes MedEd-Symposium durchgeführt, das den Perspektiven der ärztlichen Bildung gewidmet war (siehe Seite 1557 in dieser Ausgabe der Schweizerischen Ärztezeitung). Fast zweihundert Teilnehmer nutzten die Gelegenheit, sich mit den Herausforderungen und Konsequenzen auseinanderzusetzen, mit denen die ärztliche Bildung konfrontiert sein wird. Die Strukturen der Weiterbildungsstätten, die Methoden in vielen Fachdisziplinen und die Rahmenbedingungen innerhalb des Gesundheitswesens sind im Wandel und werden sich weiter ändern. Den Verantwortlichen stellt sich damit die Aufgabe, allenfalls notwendige Massnahmen nicht erst unter dem Druck bereits eingetretener Zwangslagen zu ergreifen, sondern die sich abzeichnenden Entwicklungen jetzt zu analysieren und darauf zu reagieren.

In Referaten und Seminaren wurden verschiedene Brennpunkte aufgegriffen und als mehr oder weniger grosse Baustellen beschrieben:

- Vielen Spitälern ist die Facharztweiterbildung ein echtes Anliegen. Sie erkennen ihre enorme Bedeutung für die Zukunft und sind bereit, ihren Beitrag dazu zu leisten. Leider trifft dies aber nicht für alle Häuser zu. Da und dort bekommt man zu hören, dass das Leben mit Spitalfachärzten und ohne Weiterzubildende einfacher und der Klinikbetrieb produktiver wären (Anmerkung des Setzers: kurzsichtig).

\section{Weiterbildung ist essentiell. Ihre Finanzierung muss gewährleistet sein.}

- Zum Missmut im Spitalalltag trägt neben den administrativen Verpflichtungen und dem ökonomischem Druck auch die als ungenügend empfundene Finanzierung der Weiterbildung bei. Überhaupt nicht nachvollziehbar sind die unterschiedlichen kantonalen Beiträge an die Weiterbildung und die noch immer nicht zustande gekommene Vereinbarung über interkantonale Ausgleichszahlungen.

- Im stationären und im ambulanten Sektor kommt es zunehmend zum Umbau von Strukturen. Neben den traditionellen Fachkliniken und -ambulatorien entstehen neue organisatorische Bereiche und Zentren, in denen oft meh- rere Disziplinen vertreten sind (Beispiele: Wirbelsäulenoder Gefässzentren). Bei der Planung solcher Projekte sind einerseits die Anforderungen der Weiterbildung mit zu berücksichtigen. Andererseits müssen wir diesen Entwicklungen auch in den Weiterbildungsreglementen Rechnung tragen und dürfen innovative Projekte nicht behindern.

\section{Trotz bürokratischen und administra- tiven Belastungen: Die Weiterbildung muss Kernaufgabe bleiben.}

- Wie gelingt es, in Zukunft die richtigen Fachärzte zum richtigen Zeitpunkt in der richtigen Anzahl am richtigen Ort praktizieren zu lassen und sie im Rahmen der Weiterbildung richtig darauf vorzubereiten? Diese komplexe Frage wird vor allem von Exponenten der Politik und der Gesundheitsadministration immer hörbarer gestellt. Keinesfalls kann sie mit irgendeinem rigiden Steuerungs- oder Planungsinstrument beantwortet werden. Die Abschätzung des Bedarfs eines bestimmten ärztlichen Angebots und der Entwicklung von Arbeitsmodellen, medizinischen Methoden und Berufsbildern auf sechs und mehr Jahre hinaus kann höchstens approximativ erfolgen. Eine Einflussnahme auf das System müsste deshalb in jedem Fall sehr flexibel, transparent und am ehesten anreizorientiert geschehen.

- Die Fachliteratur liefert uns immer wieder Abkürzungen, bei denen es zu evaluieren gilt, ob publizistische Eintagsfliegen oder Begriffe dahinterstecken, mit denen ernst zu nehmende Entwicklungen beschrieben werden. Im Gespräch sind EPA und CPD: EPA heisst «entrusted professional activity» und meint eine Umkrempelung der Lernzieldefinition in Richtung der Beschreibung integrierter Kompetenzen. CPD bedeutet «continuing professional development» und meint die Erweiterung der Fortbildung hin zu einer lebenslangen Weiterentwicklung aller Aspekte der Arztpersönlichkeit.

Mag sein, dass das x-te MedEd-Symposium dereinst schlüssige und erfreuliche Antworten auf die aktuellen Fragen liefern kann. Zweifellos werden dann aber neue Probleme am Horizont auftauchen. Denn wie sagte doch Osler: «The philosophies of one age have become the absurdities of the next, and the folishness of yesterday has become the wisdom of tomorrow.»

Dr. med. Werner Bauer, Präsident des Schweizerischen Instituts für ärztliche Weiter- und Fortbildung SIWF 